problem is that we have neglected the spin on the intervening oxygen. The third is that Eq. 1 describes the ground state for a single FM bond, implying that neutron scattering should be elastic, not inelastic as seen experimentally.

The first difficulty has a simple resolution. At finite hole densities, the polarization clouds overlap and the isolated impurity model is inadequate. A crude model, which considers the overlaps, simply truncates the polarization clouds at neighboring impurity sites. Because FM impurity bonds are randomly distributed, the inversion symmetry characterizing isolated impurities is broken, thus allowing intensity at $\tilde{q}=\pi$. Because we know the impurity density, $x$, from neutron activation analysis, the only parameters in such a description are the extent of the polarization cloud, $\kappa^{-1}$, which we adjusted to optimize the fit to our data. As shown by the red lines in Fig. 4, the model provides a good account of the data with $\kappa^{-1}=8.1 \pm 0.2$, $7.3 \pm 0.2$, and $7.2 \pm 0.5$ for $x=0.04,0.095$, and 0.14 respectively. These values are close to the exponential decay length of 6.03 calculated for the AFM spin polarization at the end of an $S=1$ chain $(25)$.

The modeling described so far does not include the spins of the holes responsible for the effective FM couplings between $\mathrm{Ni}^{2+}$ ions. The holes reside in oxygen orbitals of $\mathrm{Y}_{2-x} \mathrm{Ca}_{x} \mathrm{BaNiO}_{5}$ (5) but are almost certainly not confined to single, isolated oxygens. We consequently generalized Eq. 1 to take into account the hole spins, with-for the sake of definiteness - the same net amplitude as either of the $\mathrm{Ni}^{2+}$ spins next to the FM bond and distributed (with exponential decay) over $\ell$ lattice sites centered on the FM bond. As long as $\ell$ exceeds the modest value of 2 , comparable to the localization length deduced from transport data (5), the pronounced asymmetry about $\pi$ that occurs when $\ell=0$ is relieved sufficiently to produce fits indistinguishable from those in Fig. 4.

How do we account for the inelasticity of the incommensurate signal? One approach is to view the chain as consisting not of the original $S=1$ degrees of freedom but of the composite spin degrees of freedom induced around holes. The latter interact through overlapping AFM polarization clouds and hole wave functions, to produce effective couplings of random sign because the impurity spacing can be even or odd multiples of the Ni-Ni separation. With weak interchain coupling, the ground state is likely to be a spin glass, as deduced from other experiments (10) on $\mathrm{Y}_{2-x} \mathrm{Ca}_{x} \mathrm{BaNiO}_{5}$. The "incommensurate" nature of the excitations continues to follow from the structure factor of the spin part of the hole wave functions.

In summary, we have measured the magnetic fluctuations in single crystals of a doped one-dimensional spin liquid. At energies above the spin gap, the triplet excitations of the parent compound, $\mathrm{Y}_{2} \mathrm{BaNiO}_{5}$, persist with doping. However, below the gap, we find new excitations with a broad spectrum and characteristic wave vectors that are displaced from the zone boundary by an amount of order the inverse correlation length for the parent. The incommensurate fluctuations, encountered here in a doped one-dimensional transition metal oxide, resemble those seen in metallic cuprates. However, one-dimensionality makes them easier to model for $\mathrm{Y}_{2} \mathrm{BaNiO}_{5}$, and our analysis reveals that "incommensurate" peaks arise naturally even without hole order because of the characteristic spin density modulation that develops around a defect in the singlet ground state of a quantum spin liquid. This phenomenon accounts for the weak dependence of the incommensurability on doping in the one-dimensional nickelate. Indeed, $\mathrm{Y}_{2-x} \mathrm{Ca}_{x} \mathrm{BaNiO}_{5}$ gives us a quantitative impression of the magnetic polarization cloud associated with the holes in a doped transition metal oxide. Our results imply that the spin part of the hole wave function is actually the edge state nucleated by the hole in a quantum spin fluid.

\section{References and Notes}

1. S.-W. Cheong et al., Phys. Rev. Lett. 67, 1791 (1991).

2. S. M. Hayden et al., Phys. Rev. Lett. 68, 1061 (1992).

3. H. A. Mook et al., Nature 395, 580 (1998).

4. J. M. Tranquada, B. J. Sternlieb, J. D. Axe, Y. Nakamura, S. Uchida, Nature 375, 561 (1995).

5. J. F. DiTusa et al., Phys. Rev. Lett. 73, 1857 (1994).

6. J. Darriet and L. P. Regnault, Solid State Commun. 86, 409 (1993)
7. J. F. DiTusa et al., Physica B 194-196, 181 (1994).

8. G. Xu et al., Phys. Rev. B 54, R6827 (1996).

9. T. Sakaguchi, K. Kakurai, T. Yokoo, J. Akimitsu, Phys. Soc. Jpn. 65, 3025 (1996).

10. K. Kojima et al., Phys. Rev. Lett. 74, 3471 (1995).

11. E. Dagotto, J. Riera, A. Sandvik, A. Moreo, Phys. Rev. Lett. 76, 1731 (1996).

12. Z.-Y. Lu, Z.-B. Su, L. Yu, Phys. Rev. Lett. 74, 4297 (1995).

13. K. Penc and H. Shiba, Phys. Rev. B 52, R715 (1995).

14. S. Fujimoto and N. Kawakami, Phys. Rev. B 52, 6189 (1995).

15. E. S. Sorensen and I. Affleck, Phys. Rev. B 51, 16115 (1995).

16. R. A. Hyman, K. Yang, R. N. Bhatt, S. M. Girvin, Phys. Rev. Lett. 76, 839 (1996).

17. I. Affleck, T. Kennedy, E. H. Lieb, H. Tasaki, Phys. Rev. Lett. 59, 799 (1987).

18. T. Kennedy, J. Phys. Condens. Matter 2, 5737 (1990).

19. F. D. M. Haldane, Phys. Lett. 93A, 464 (1983).

20. C. H. Chen, S.-W. Cheong, A. S. Cooper, Phys. Rev. Lett. 71, 2461 (1993).

21. J. M. Tranquada, D. J. Buttrey, V. Sachan, J. E. Lorenzo, Phys. Rev. Lett. 73, 1003 (1994).

22. S.-H. Lee and S.-W. Cheong, Phys. Rev. Lett. 79, 2514 (1997).

23. V. J. Emery and G. Reiter, Phys. Rev. B 38, 4547 (1988).

24. A. Aharony et al., Phys. Rev. Lett. 60, 1330 (1988).

25. S. R. White and D. A. Huse, Phys. Rev. B 48, 3844 (1993).

26. We thank T. M. Rice, A. J. Millis, and Q. Huang for useful discussions and A. Krishnan for transmission electron microscopy measurements. Work at Johns Hopkins University was supported by the NSF under DMR-9453362. Work at Louisiana State University was supported by the NSF under DMR-9702690 and the State of Louisiana Board of Regents under LEQSF(RF/1996-99)-RD-A05. This work used neutron research facilities supported by the National Institute of Standards and Technology (NIST) and the NSF through DMR-9423101.

2 March 2000; accepted 25 May 2000

\title{
Subatomic Features on the Silicon (111)-(7×7) Surface Observed by Atomic Force Microscopy
}

\section{Franz J. Giessibl,* S. Hembacher, H. Bielefeldt, J. Mannhart}

\begin{abstract}
The atomic force microscope images surfaces by sensing the forces between a sharp tip and a sample. If the tip-sample interaction is dominated by short-range forces due to the formation of covalent bonds, the image of an individual atom should reflect the angular symmetry of the interaction. Here, we report on a distinct substructure in the images of individual adatoms on silicon (111)$(7 \times 7)$, two crescents with a spherical envelope. The crescents are interpreted as images of two atomic orbitals of the front atom of the tip. Key for the observation of these subatomic features is a force-detection scheme with superior noise performance and enhanced sensitivity to short-range forces.
\end{abstract}

In atomic force microscopy (AFM) (1), surfaces are imaged with atomic resolution by bringing a probe into close proximity and

Experimentalphysik VI, Center for Electronic Correlations and Magnetism (EKM), Institute of Physics, University of Augsburg, 86135 Augsburg, Germany.

*To whom correspondence should be addressed. Email: franz.giessibl@physik.uni-augsburg.de sensing the interaction force while scanning the surface. It has been shown that a dynamic mode of imaging is required for resolving reactive surfaces like $\mathrm{Si}(111)$ in an ultrahigh vacuum where chemical bonding between a tip and a sample can occur $(2,3)$. Frequencymodulation AFM (FM-AFM) (4) enables fast imaging in a vacuum and is now the commonly used imaging technique. In FM-AFM, 
a cantilever beam with eigenfrequency $f_{0}$ and spring constant $k$ holding a sharp tip at its free end oscillates with a constant amplitude $A$. When this cantilever is brought close to a sample, the tip-sample forces cause a frequency shift $\Delta f$, which is used as the imaging signal. The relevant imaging parameter in FM-AFM is the "normalized frequency shift" $\gamma=\Delta f k A^{3 / 2} / f_{0}$, when $A$ is large in comparison to the range of the tip-sample forces (5).

A recent study has shown that the optimal signal-to-noise ratio is obtained for amplitudes roughly equal to the range of interatomic forces, much smaller than the currently used values of $A \sim 10 \mathrm{~nm}(6)$. Stable operation at small amplitudes is, however, only possible if the cantilever is sufficiently stiff (5). An additional advantage of small amplitudes is the reduced sensitivity to long-range forces and the enhanced sensitivity to shortrange forces (7). Unprecedented spatial resolution has been observed with a force sensor designed in accordance with these findings (8). This sensor was used in the present study.

Because of its complexity, the Si(111)$(7 \times 7)$ surface (Fig. 1) has been an intriguing object of study to many surface scientists. After Binnig et al. succeeded in imaging the top atom layer of $\mathrm{Si}(111)-(7 \times 7)$ in their landmark experiments by scanning tunneling microscopy (STM) (9), Takayanagi et al. developed the dimer-adatom-stacking fault (DAS) model (10), which is now commonly accepted. According to this model (Fig. 1, A and B), six adatoms are located in each half of the unit cell. The adatoms are bound by covalent bonds formed by $3 \mathrm{sp}^{3}$ hybrid orbitals. One of the four $3 \mathrm{sp}^{3}$ hybrid orbitals is pointing perpendicular to the surface and forms a dangling bond. Brommer et al. (11) have computed the coordinates of all the atoms in the $7 \times 7$ unit cell by molecular dynamics calculations and have found that the four inequivalent adatom sites have a different height (Fig. 1B).

In 1995, the $\operatorname{Si}(111)-(7 \times 7)$ surface was first imaged with FM-AFM $(2,3)$. These FM-AFM images looked similar to the STM images acquired at positive sample bias (empty states). It has been determined that the formation of a chemical bond between the front atom of the tip and the adatoms is responsible for the experimentally observed atomic contrast (12).

In the present work, the $\operatorname{Si}(111)-(7 \times 7)$ surface was investigated using FM-AFM with optimized resolution. The microscope is a homebuilt scanning tunneling microscope/atomic force microscope based on a commercial scanning tunneling microscope (ThermoMicroscopes, Sunnyvale, California). The electronics operating the force sensor is also homebuilt, relying on a commercial frequency demodulator (Nanosurf AG, Liestal, Switzerland). The vacuum chamber, pumped with an ion and titanium sublimation pump, has a base pressure of $5 \times$ $10^{-11}$ mbar. An $11 \mathrm{~mm}$ by $14 \mathrm{~mm}$ piece of a boron-doped (p-type) $\mathrm{Si}(111)$ wafer was used as a sample, and the $7 \times 7$ reconstruction was obtained by heating the sample to $1300^{\circ} \mathrm{C}$ for $30 \mathrm{~s}$ with an electron beam heater while keeping the pressure during heating below $5 \times 10^{-9}$ mbar. The force sensor, described in detail in (8), is made of a quartz tuning fork with an etched tungsten tip attached to it. The spring constant of the sensor was $k=1800 \mathrm{~N} / \mathrm{m}$, the eigenfrequency was $f_{0}=16,860 \mathrm{~Hz}$, and all data were recorded with an amplitude $A=0.8 \mathrm{~nm}$.

The experiment was started by operating the microscope in the STM mode, with the feedback being controlled by the tunneling current. A tunneling voltage of $+1.6 \mathrm{~V}$ was applied to the oscillating tip. The tunneling current was collected at the sample, and the set point was adjusted to $60 \mathrm{pA}$. The surface was scanned to find a clean spot that displayed large areas of reconstructed silicon. Also, controlled collisions between the tip and sample were performed to shape the tip so that good STM images were obtained. The corresponding frequency shift was approximately $-150 \mathrm{~Hz}$. These STM images showed only a single maximum per adatom. Next, the tip was withdrawn, and the feedback was switched to frequency-shift control. As the set point of the frequency shift was slowly decreased while the tip was scanning, the corrugation of the image was increased until an optimal value was reached.

Figure 2 shows a panel of consecutively recorded topographic images (obtained at constant frequency shift) of the silicon surface. The sequence from Fig. 2A to $2 \mathrm{~B}$ shows that the window for the frequency shift where optimal resolution is obtained is rather small. Three features of Fig. 2, B to D, are quite remarkable: the adatoms display a distinct substructure, with the image of each adatom composed of two crescents; the corner holes are very deep in comparison to previous AFM images $(\sim 180 \mathrm{pm})$; and the vertical resolution is excellent, such that the height differences of the four distinct types of adatoms can be measured (Fig. 1, C and D). The ranking in the experimental height of the adatoms is equal for FM-AFM and low-energy electron diffraction (LEED) data: 1 is the highest, and 2, 4, and 3 follow, with 3 being the lowest. However, the height differences measured by FM-AFM are about three times as large as the values measured by LEED and four to seven times as large as the values of the theoretical data (11). The discrepancy in magnitude between the LEED and the FM-AFM data is probably due to elastic deformations of the tip and sample. The mean height of the adatoms in the left
Fig. 1. (A) Top view and (B) side view of the DAS model of Si(111)(7×7) (11). Adatoms and rest atoms are emphasized by large and small black spheres, respectively. The left half of the unit cell has a stacking fault; the right half is unfaulted. The 12 adatoms within one unit cell belong to four different classes. The three adatoms within one class are related by symmetry operations (rotation by $2 \pi / 3$ ). The theoretical equilibrium positions of adatoms 1 , 2 , and 4 are $8.5,3.1$, and $3.8 \mathrm{pm}$ higher than adatom 3, respectively, whereas the height differences as measured by LEED are 12, 8 , and 4 pm, respectively (11). (C) FM-AFM image (raw data) of the $\mathrm{Si}(111)-(7 \times 7)$ unit cell (section of Fig.
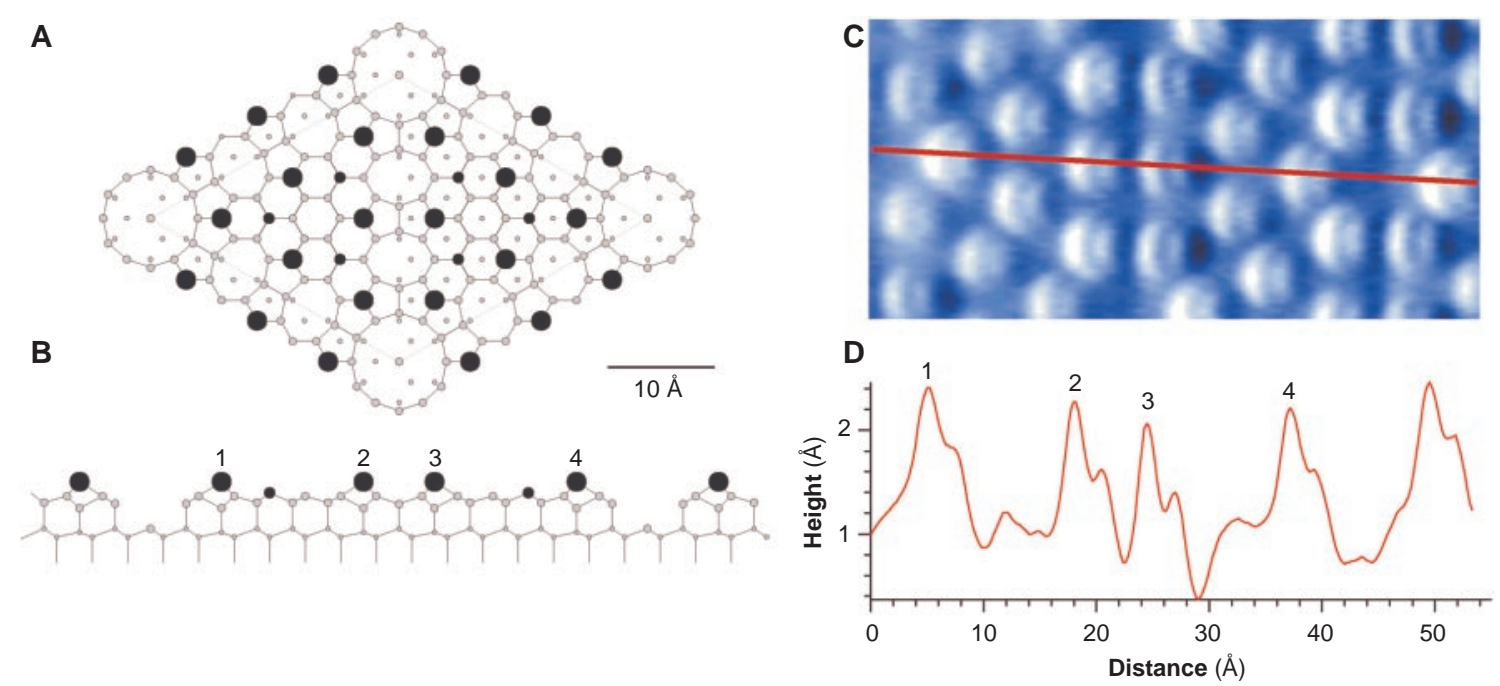

2B, left). (D) Profile of (C). Adatoms 1, 2, and 4 are 34, 19, and 15 pm higher than adatom 3 , respectively. 


\section{R E P O R T S}

and right lower image sections of Fig. 2D (acquired at $\Delta f=-140 \mathrm{~Hz}$ ) is only $18 \mathrm{pm}$ greater than the mean height in the upper image sections (acquired at $\Delta f=-160 \mathrm{~Hz}$ ). The observation that the image contrast changes substantially by such a small change in the minimal tip-sample distance leads to the conclusion that the minimal tip-sample distance is of the order of the next-neighbor distances in the bulk and is much smaller than previously estimated (2).

At first glance, the double-peaked adatoms look like a typical double-tip image. However, in a common double-tip image, the distance between the two subimages has to be at least as large as the next-neighbor distance in the tip material, which is $316 \mathrm{pm}$ for tungsten (13), whereas the experimental distance between the observed maxima is only $220 \mathrm{pm}$. If the tip had two "mini tips" interacting with the sample, where the two tips are displaced by $220 \mathrm{pm}$ plus a surface lattice vector, the atomic defects in the left upper sections of Fig. 2, B to D, should appear again on a different location in the image, displaced by roughly one lattice vector. Furthermore, an image obtained by a superposition of two closely spaced single-tip images would yield an elliptical envelope of the adatom image, whereas the envelope of the experimental images is spherical.

The nominal depth of the corner holes is $445 \mathrm{pm}$ (11), but in typical STM experiments, a depth of $200 \mathrm{pm}$ is observed. The first AFM image of $\operatorname{Si}(111)-(7 \times 7)$ was recorded with $A=$ $34 \mathrm{~nm}$, and the corner-hole depth was $73 \mathrm{pm}$ (2), suggesting that AFM is sensitive to both short- and long-range interactions. The influence of the long-range interactions causes the contrast to decrease (7). Lantz et al. have imaged $\mathrm{Si}(111)-(7 \times 7)$ at low temperatures with $A=5 \mathrm{~nm}$ (14) and found a corner-hole depth of $\sim 120 \mathrm{pm}$, whereas Uchihashi et al. (15) have measured a depth of $\sim 170$ pm with $A=$ $16 \mathrm{~nm}$. Here, we found a depth of $180 \mathrm{pm}$ with $A=0.8 \mathrm{~nm}$, which confirms our theoretical findings that smaller amplitudes reduce the sensitivity to long-range forces and enhance the image contrast (7).

For further analysis, the structure of a sin- gle-adatom image was studied (Fig. 3 shows a magnification of Fig. 2B). Because the structural and electronic arrangements of silicon are well known and a single dangling bond per adatom is predicted, the split adatom must be caused by the tip, according to the reciprocity principle (16). For an explanation of this adatom image, we first looked at the electronic structure of tungsten. Tungsten condenses in a body-centered cubic structure where each atom has eight next neighbors. According to Pauling (17), metals also display a partly covalent binding character. Assuming that the tip-sample interaction is formed by covalent bonds between tungsten and the $3 \mathrm{sp}^{3}$ hybrid orbitals of the silicon adatom, three or four maxima (depending on the orientation of the tungsten tip cluster) would be expected for each adatom (18). Thus, the experimental image cannot have been created with a tungsten tip. However, experimental evidence has been found in STM where "controlled collisions" between a tungsten tip and silicon lead to picking up a silicon cluster from the sample $(19,20)$. Because the planes with the lowest surface energy in silicon

Fig. 2. Series of topographic images of Si $(111)-(7 \times 7)$ observed by FM-AFM. The fast scanning direction was from left to right for the left side and from right to left for the right side. All images are raw data, except for the background plane subtraction. All images were recorded with a scanning speed of four lines per second in the horizontal direction, and the image size is 256 by 256 pixels; thus, the acquisition time for one frame is $64 \mathrm{~s}$. The tip bias was maintained at $+1.6 \mathrm{~V}$ with respect to the sample. The direction of the slow scan was reversed after an image was completed; in $(A),(C)$, and (E), the slow scan direction was from top to bottom, and in (B), (D), and $(F)$, the direction was from bottom to top. (A) Frequency shift $\Delta f=-130 \mathrm{~Hz}$, weak contrast. (B)
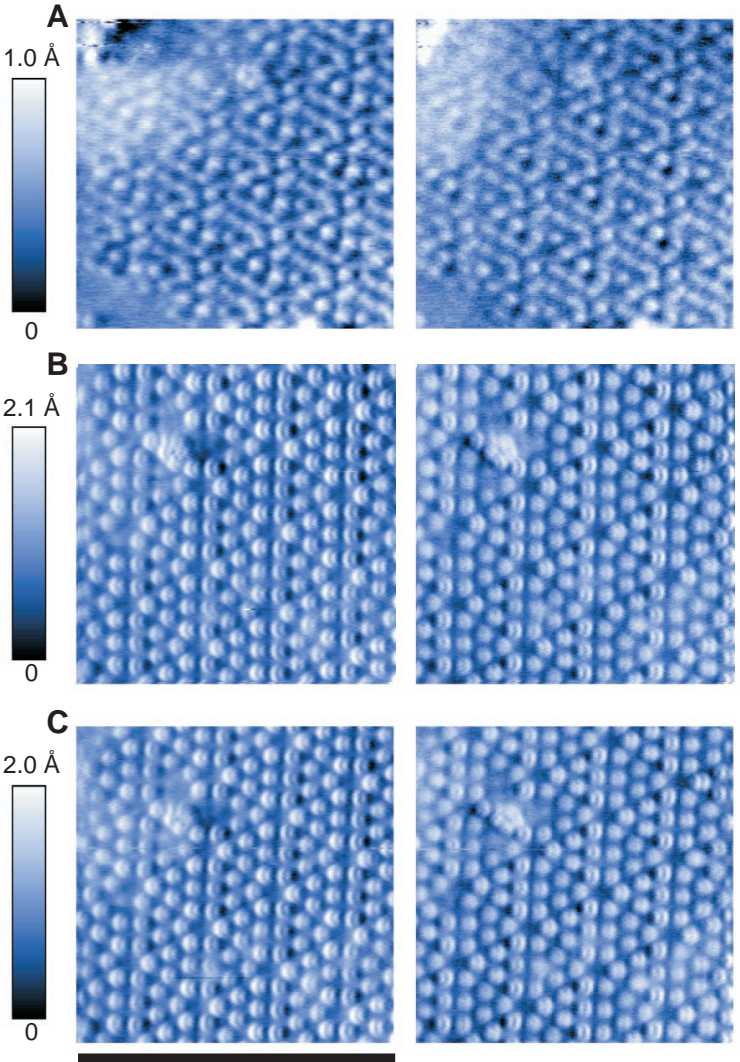
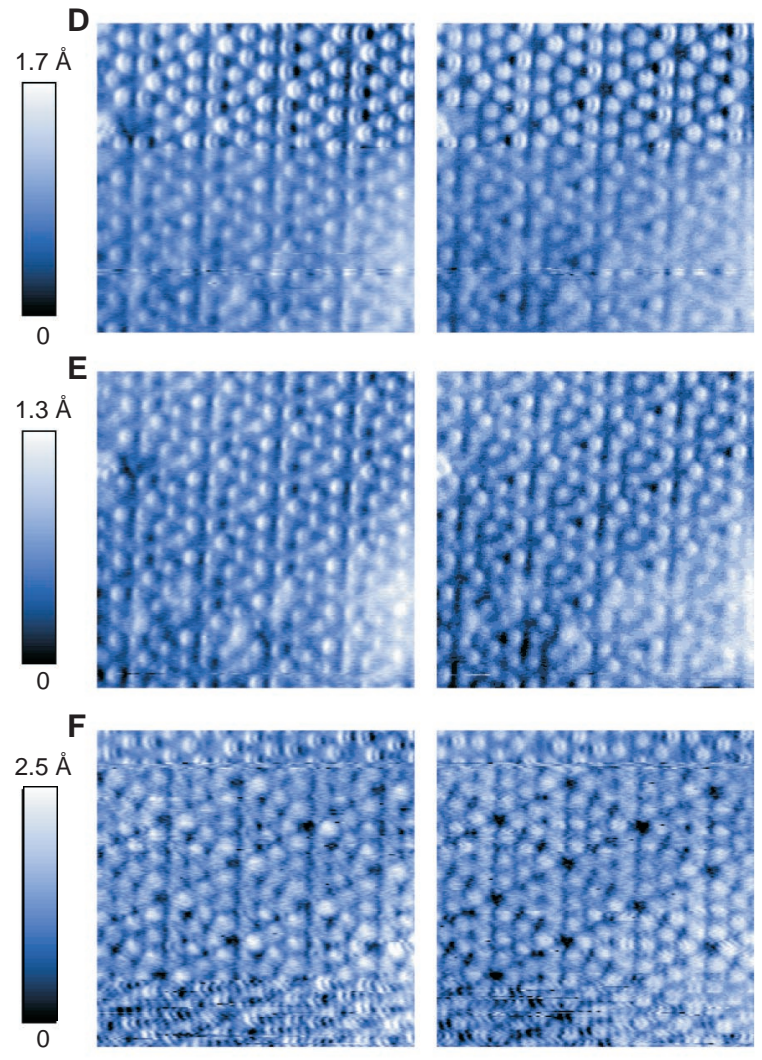
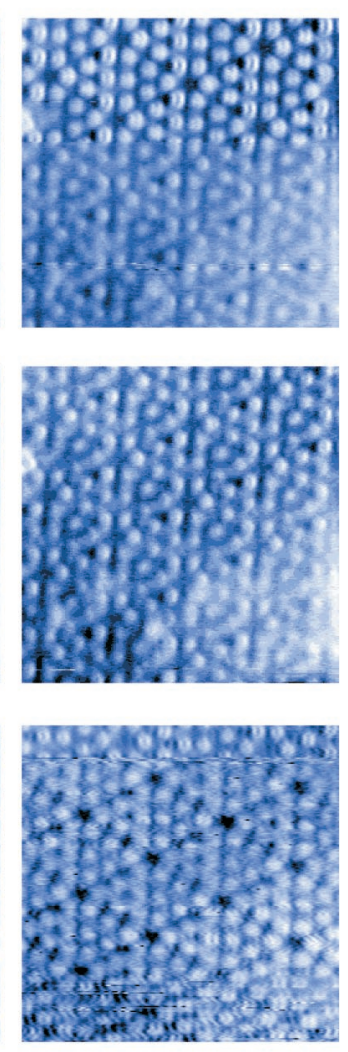

$100 \AA$

$\Delta f=-160 \mathrm{~Hz}$, optimal contrast. A defect is seen in the left upper section of the image, and there are dual maxima for each adatom. (C) $\Delta f=-160$ $\mathrm{Hz}$, image similar to (B). (D) Before the start of this scan, the tip was displaced to the right by a distance of $2 \mathrm{~nm}$; consequently, the defect appears shifted to the left by a distance of $2 \mathrm{~nm}$. After 102 scan lines were completed, the set point of the frequency shift was set to $\Delta f=-140 \mathrm{~Hz}$. From then on, each adatom showed only one maximum, the contrast decreased, and the overall noise increased. Because the lateral positions

of the atoms are not shifted, the same tip atom is active in the lower and upper sections of the image. (E) $\Delta f=-140 \mathrm{~Hz}$, image comparable to lower section of (D). (F) $\Delta f=-160 \mathrm{~Hz}$. The noise in the upper 35 scan lines was very low again. However, at scan line 35 , the tip has apparently collapsed. This is evident because of the increased noise and a shift in the image pattern by $\sim 1 \mathrm{~nm}$ to the lower right. After this tip change, excellent imaging conditions could not be retained with this tip. 
are (111) planes, the corner atoms of a silicon cluster with minimal surface energy expose two dangling bonds. If a silicon cluster on top of a tungsten tip exposes a sharp tip perpendicular to the sample plane, the front atom has two dangling bonds interacting with the single dangling bond of the adatom.

Stillinger and Weber (SW) have found an eight-parameter semiempirical analytic model potential (21) that describes the nearest and next nearest neighbor interaction in silicon. The parameters are fitted so that the bulk properties of silicon are modeled very well for both the solid and the liquid phase at high and low pressure, that is, up to large interatomic distances that exist in the case of AFM where chemical bonds are stretched up to the disintegration of the bond. The normalized frequency shift $\gamma$ was calculated in Fig. 4, B and C, from the SW potential according to equation 12 in (7). The use of the largeamplitude approximation for $\gamma$ is justified (even with $A=0.8 \mathrm{~nm}$, the ratio between $A$ and the range of the SW potential is $\sim 10$ ), as shown by figure 2 in (7).

Figure 4A shows a tip consisting of a silicon cluster pointed in a $<100>$ direction interacting with an adatom on $\mathrm{Si}(111)$ $(7 \times 7)$. Figure $4 \mathrm{~B}$ is a calculation of the normalized frequency shift $\gamma$ in an $x y$ plane that is elevated at $z=310 \mathrm{pm}$ above the plane connecting the centers of the adatoms. One maximum appears at the adatom positions. When $z$ is reduced to $285 \mathrm{pm}$, a double maximum appears (Fig. 4C). Figure $2, \mathrm{~B}$ and $\mathrm{C}$, is in good agreement with Fig. $4 \mathrm{C}$, and the transition from Fig. $4 \mathrm{~B}$ to $4 \mathrm{C}$ is experimentally found in Fig. 2D. Figure 4D shows the calculated adatom image when the tip is tilted around the $y$ axis by $3^{\circ}$. The theoretical distance of the double peaks is $\sim 230 \mathrm{pm}$, and the experimentally found distance is $\sim 220 \mathrm{pm}$. The reduction of the double maxima at $z=285 \mathrm{pm}$ to a single maximum at $z=310 \mathrm{pm}$ (in a ball-andstick model of the covalent bond, the dis-

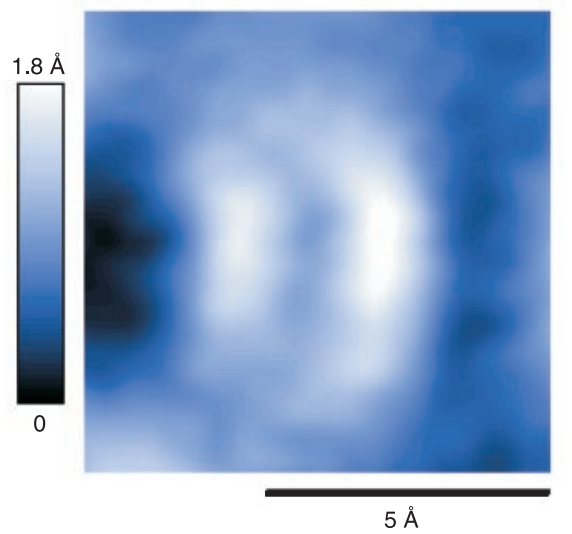

Fig. 3. Enlargement of a single adatom image (from Fig. 2B, right).

tance of the two maxima would increase for increasing $z$ ) is not an idiosyncrasy of the SW potential. The chemical bond between the tip and sample can also be described as an overlap of atomic orbitals. A simple calculation of the charge density of two $3 \mathrm{sp}^{3}$ orbitals with an orientation like the tip orbitals in Fig. 4A using hydrogen-like

A
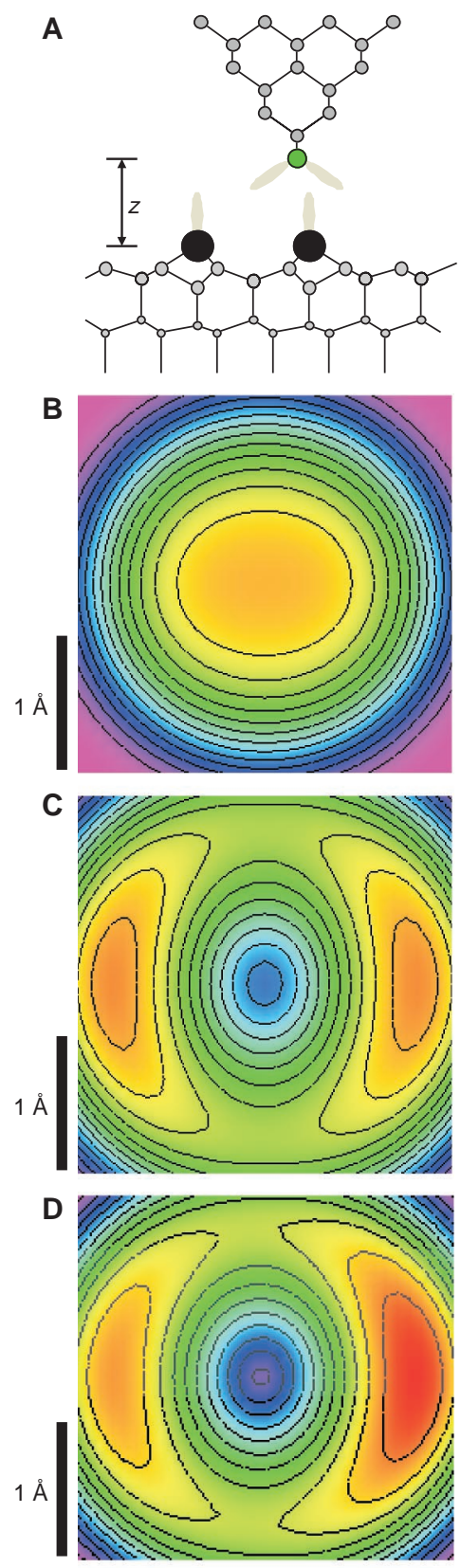

$-8 \mathrm{fN} \sqrt{\mathrm{m}}$

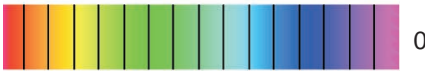

Fig. 4. (A) Schematic drawing of the geometry used for the calculation, sketching the $3 \mathrm{sp}^{3}$ hybrid orbitals of the tip atom (green sphere) and sample adatoms (black spheres). Simulated $2.8 \AA$ by $2.8 \AA$ constant height images (normalized frequency shift) of a single adatom for (B) $z=310 \mathrm{pm},(\mathbf{C}) \mathrm{z}=285 \mathrm{pm}$, and (D) $z=285$ $\mathrm{pm}$ with the tip tilted $3^{\circ}$ around the $y$ axis. wave functions yields a similar result. A slight deviation between theory and experiment is noted, however. The simulated picture in Fig. 4C shows two crescents facing each other, whereas the experimentally observed crescents in Fig. 2 open to the right for the scan directions from left to right and open to the left with scan directions from right to left. Also, within each pair of crescents corresponding to one adatom, the left crescents are higher than the right ones for the scans from left to right; the crescents appear to be about equally high for the scan from right to left. This height difference suggests that the two tip orbitals were not oriented perfectly symmetrical with respect to the sample normal vector but were slightly tilted, as simulated in Fig. 4D. The asymmetry in images scanned from left to right and from right to left suggest the presence of either minor feedback errors or small elastic deformations of the tip and sample. However, a detailed analysis of the feedback parameters and more experiments in a constant height mode have confirmed the main observation of subatomic structure by AFM (18).

\section{References And Notes}

1. G. Binnig, C. F. Quate, Ch. Gerber, Phys. Rev. Lett. 56, 930 (1986).

2. F. J. Giessibl, Science 267, 68 (1995).

3. S. Kitamura and M. Iwatsuki, Jpn. J. Appl. Phys. 34, L145 (1995).

4. T. R. Albrecht, P. Grütter, D. Horne, D. Rugar, J. Appl. Phys. 69, 668 (1991).

5. F. J. Giessibl, Phys. Rev. B 56, 16010 (1997).

6. , H. Bielefeldt, S. Hembacher, J. Mannhart, Appl. Surf. Sci. 140, 352 (1999).

7. F. J. Giessibl and H. Bielefeldt, Phys. Rev. B 61, 9968 (2000).

8. F. J. Giessibl, Appl. Phys. Lett. 76, 1470 (2000).

9. G. Binnig, H. Rohrer, C. Gerber, E. Weibel, Phys. Rev. Lett. 50, 120 (1983).

10. K. Takayanagi, Y. Tanishiro, S. Takahashi, J. Vac. Sci. Technol. A 3, 1502 (1985).

11. K. D. Brommer, M. Needels, B. E. Larson, J. D. Joannoupoulos, Phys. Rev. Lett. 68, 1355 (1992).

12. R. Perez, I. Stich, M. C. Payne, K. Terakura, Phys. Rev. Lett. 78, 10835 (1997).

13. N. Ashcroft and N. D. Mermin, Solid State Physics (Saunders, Philadelphia, 1981).

14. M. A. Lantz et al., Phys. Rev. Lett. 84, 2642 (2000).

15. T. Uchihashi et al., Phys. Rev. B 56, 9834 (1997).

16. C. J. Chen, Introduction to Scanning Tunneling Microscopy (Oxford Univ. Press, New York, 1993).

17. L. Pauling, The Nature of the Chemical Bond (Cornell Univ. Press, Ithaca, NY, ed. 2, 1957).

18. In another experiment, using a metal tip, we observed images of adatoms that displayed a fourfold symmetry; see supplemental material, available at www. sciencemag.org/feature/data/1050833.shl.

19. C. J. Chen, J. Vac. Sci. Technol. A 9, 44 (1991).

20. J. E. Demuth, U. Köhler, R. J. Hamers, J. Microsc. 151, 299 (1988).

21. F. H. Stillinger and T. A. Weber, Phys. Rev. B 31, 5262 (1985).

22. We thank D. Schlom for discussions, K. Wiedenmann for technical assistance, and Nanosurf AG for supplying components of the force sensor and a phaselocked-loop frequency demodulator. This work is supported by the Bundesministerium für Bildung, Wissenschaft, Forschung und Technologie (project number 13N6918/1).

28 March 2000; accepted 20 June 2000 


\section{Science \\ MIAAAS}

Subatomic Features on the Silicon (111)-(7×7) Surface Observed by Atomic Force Microscopy

Franz J. Giessibl, S. Hembacher, H. Bielefeldt and J. Mannhart (July 21, 2000)

Science 289 (5478), 422-425. [doi: 10.1126/science.289.5478.422]

Editor's Summary

This copy is for your personal, non-commercial use only.

Article Tools Visit the online version of this article to access the personalization and article tools:

http://science.sciencemag.org/content/289/5478/422

Permissions Obtain information about reproducing this article: http://www.sciencemag.org/about/permissions.dtl

Science (print ISSN 0036-8075; online ISSN 1095-9203) is published weekly, except the last week in December, by the American Association for the Advancement of Science, 1200 New York Avenue NW, Washington, DC 20005. Copyright 2016 by the American Association for the Advancement of Science; all rights reserved. The title Science is a registered trademark of AAAS. 